\title{
Treatment Strategies for Multiple Antimicrobial-Resistant Bacterial Infections
}

\section{Deniz Ozkaya*}

Department of Infectious Diseases and Clinical Microbiology, Turkish Health Ministry, Cigili Education and Research Hospital, Turks and Caicos Islands

\section{Editorial}

Acinetobacter baumannii is a major pathogen of health care associated infections (HCAI) throughout the world. Acinetobacter is located in front row with persistence in the hospital environment, with its high mortality and morbidity development potential, and transfer of resistance genes between bacteria and other species [1]. Carbapenemase activity is the most important cause of carbapenem resistance; therefore, all efforts to develop carbapenemase inhibitory agents derive from the aim of carbapenem resistance prevention for $A$. baumannii and other Gram-negative enteric bacteria [2]. According to Antimicrobial Surveillance Program (SENTRY) reports, carbapenem resistance rates among $A$. baumannii isolated from two Turkish Health Centers were reported between 20-60\% [3]. Although carbapenem resistance in A. baumannii strains in Turkey was seen sporadically until 2005, isolation rates have increased since August 2005 [4]. Many outbreaks related to multidrug-resistant $A$. baumannii have been reported in Turkish hospitals as a result of molecular epidemiologic studies in recent years [5,6]. Major outbreaks were caused by OXACarbapenemases. The first outbreak appeared with OXA-23 and OXA-48 carbapenemases-producing A. baumannii between 2005 and 2006 [7]. The outbreaks continued in the following years and MDR Acinetobacter has been established as an endemic strain in hospitals. All these have led to a further increase of resistance as well as more carbapenem use. The widespread use of carbapenem in the empirical treatment of $A$. baumannii infections has also begun to restrict the use of the carbapenem for the treatment of other Gram-negative enteric bacterial infections.

The importance of sulbactam-containing antibiotics has increased in the empiric treatment of $A$. baumannii infections. In addition, colistin, cefoperazone sulbactam, and tigecycline began to be preferred for the treatment of such infections. In accordance with Antibacterial Stewardship Policy, the policy of existing antibiotics requires the rational use of antibiotics in accordance with appropriate indications.

Apart from Acinetobacter and carbapenem-resistant Enterobacteriaceae strains, OXA-48 carbapenemase-producing bacteria have also been reported [8]. Although these strains have been appearing periodically since 2001 , the first OXA- 48 producing bacterium was isolated from Istanbul in 2003 [9]. This bacterium was resistant to all beta-lactam antibiotics including carbapenems. Outbreaks were controlled in most cities in Turkey with effective infection control policies [10]. Today, the spread of carbapenem-producing strains is not only a problem in Turkey, but also in the Middle East, North Africa, and Europe [11]. OXA-48 was also found in C. freundi, P. retgeri, E. cloacae and E. coli strains [12,13]. The number of OXA48-producing nosocomial bacteria isolates was 6 until 2008 in our country, and increased to 27 in 2009 . However, metallobeta-lactamase activity remained stable for three years [14]. Apart from the $b l a_{\text {OXA-48 }}$ gene region, these strains had different resistant gene regions [15]. This means that Turkey harbors more than one OXA-48-producing bacterial clone. In a study by Labarca et al. [16], it was claimed that the global spread of carbapenem- resistant clones producing ST258 and carbapenemase- 2 may be an important threat through antibacterial resistance development [16]. This situation is not yet present in our country; OX-48-induced different types of bacteria are contained in our geographic area [17]. Apart from these, NDM-1 and VIM-induced bacteria-related outbreaks are seen in Turkey and Mediterranean countries. NDI-1 induced one isolate found in a patient who came to our country from Iraq [18].

Non-carbapenem treatment options in the treatment of carbapenemase-induced Enterobacteriaceae are at the forefront. Antibacterial agents should be preferred among the treatment options, according to antibacterial susceptibility results. However, it should be noted that results of in vitro results cannot reflect true in vivo results and often develop resistance to these agents during treatment. For determining the most accurate therapeutic approach, it should be preferred to detect the minimum inhibitory concentration (MIC) points of antibacterials and to use rational antibacterial treatment. In such cases, avoiding monotherapies and preferring combination therapies is essential. However, individual MIC results are not enough to select antibiotics for the combination. In addition to MIC results, synergistic or the additive effects of antibiotics should be taken into consideration. Colistin, tigecycline, fosfomycin, and doxycycline are among the most effective antibacterials against carbapenemresistant bacteria. Piperacillin-tazobactam, sefaperazone-sulbactam, ciprofloxacin, levofloxacin, and amikacin are other antibiotics that are commonly preferred. However, the majority of antibiotics in the second group bring great risk for the development of carbapenem resistance. Even if the bacteria are found susceptible to these agents in disk diffusion test results, their effectiveness in combination therapy should be closely monitored. Colistin-containing combination therapy is one of the most preferred regimes in the treatment of carbapenem-resistant Enterobacteriaceae infections. Colistin provides strong activity against these bacteria alone, as demonstrated in the results of colistin use as a monotherapy or at low doses. Use of low-dose colistin may cause an increase in the selective pressure of pathogens and contribute to new resistance patterns. The cumulative loads of bacteria are other factors that play a role in the acquisition and transmission of new resistance patterns. The treatment approach to reduce the cumulative burden of bacteria is very important to provide infection control and resistance control.

Colistin-resistant Enterobacteriaceae-related infections have

*Corresponding author: Deniz Ozkaya, Department of Infectious Diseases and Clinical Microbiology, Turkish Health Ministry, Cigili Education and Research Hospital, Cigili, Izmir, Turks and Caicos Islands, Tel: +90(232)3983700; E-mail: dnzozkaya@gmail.com

Received: December 14, 2016; Accepted: December 16, 2016; Published: December 23, 2016

Citation: Ozkaya D (2016) Treatment Strategies for Multiple AntimicrobialResistant Bacterial Infections. J Infect Dis Med 1: e101. doi: 10.4172/2576 1420.1000e101

Copyright: (C) 2016 Ozkaya D. This is an open-access article distributed under the terms of the Creative Commons Attribution License, which permits unrestricted use, distribution, and reproduction in any medium, provided the original author and source are credited. 
occurred recently in Turkey, as well as in Greece and Italy. Combination therapy should be preferred in the treatment of such infections. Other effective agents other than colistin should be evaluated. The real susceptibility of these agents can be controlled by determining MIC values. Treatment regimens can be formed with combinations that include these agents [11]. Recent studies showed that tigecycline and phosphomycin were the most commonly used agents in the treatment of colistin-resistant K. pneumoniae infections [19]. Apart from these, ertapenem- and imipenem-containing combinations are also used. Ertapenem remains non-functional due to the carbapenemase activity in this combination, which increases the effectiveness of meropenem in the target molecules. In in vitro experimental studies, it was reported that triple-agent combinations overcame carbapenem and colistin resistance problems and a synergistic effect was found. The search for different treatment, however, still continues today.

Besides the current efforts to develop new antibiotics, the dominant view is to increase the synergistic effect between different antibiotics so as to overcome the problem of resistance. Using such pharmacologic properties in the treatment of bacterial infections, there are efforts to create new combinations, continuous infusion, inhaler or intrathecal applications of drug, and double, triple or quadruple antibiotics.

Whatever new treatment strategies are used to overcome antibiotic resistance, real solutions lie in the implementation of a universal infection control policy that eliminates the colonization and settlement of antibacterial-resistant bacteria, and the dissemination of rational antibiotic use and antibiotic stewardship programs.

\section{Reference}

1. Nordmann P, Naas T, Poirel L (2011) Global spread of carbapenemaseproducing enterobacteriaceae. Emerg Infect Dis 17: 1791-1798.

2. Ozen N, Ergani A, Thierry N, Ogunc D, Gultekin M, et al. (2009) Outbreak of carbapenem-resistant acinetobacter baumannii producing the carbapenemase OXA-58 in Turkey. The Open Antimicrobial Agents Journal 1: 1-8.

3. Gur D, Korten V, Unal S, Deshpande LM, Castanheira M (2008) Increasing carbapenem resistance due to the clonal dissemination of oxacillinase (OXA23 and OXA-58)-producing acinetobacter baumannii: report from the Turkish SENTRY program sites. J Med Microbiol 57: 1529-1532.

4. Kulah C, Mooij MJ, Comert F, Aktas E, Celebi G, et al. (2010) Characterisation of carbapenem-resistant Acinetobacter baumannii outbreak strains producing OXA-58 in Turkey. Int J Antimicrob Agents 36: 114-118.

5. Vahaboglu H, Budak F, Kasap M, Gacar G, Torol S, et al. (2006) High prevalence of OXA-51-type class $D$ beta-lactamases among ceftazidime-resistant clinical isolates of Acinetobacter spp.: co-existence with OXA-58 in multiple centres. J Antimicrob Chemother 58: 537-542.

6. Alp E, Esel D, Yildiz O, Voss A, Melchers W, et al. (2006) Genotypic analysis of Acinetobacter bloodstream infection isolates in a Turkish university hospital. Scand J Infect Dis 38: 335-340.

7. Meric M, Kasap M, Gacar G, Budak F, Dundar D, et al. (2008) Emergence and spread of carbapenem-resistant Acinetobacter baumannii in a tertiary care hospital in Turkey. FEMS Microbiol Let 282: 214-218.

8. Abdullah K, Zerin A, Orhan B, Ramazan G, Yasemin B, et al. (2011) Identification and characterization of OXA-48 producing,-carbapenem-resistant enterobacteriaceae Isolates in Turkey. Ann Clin Lab Sci 41: 161-166.

9. Poirel L, Heritier C, Tolun V, Nordmann P (2004) Emergence of oxacillinasemediated resistance to imipenem in Klebsiella pneumoniae. Antimicrob Agents Chemother 48: 15-22.

10. Carrer A, Poirel L, Eraksoy H, Cagatay AA, Badur S, et al. (2008) Spread of OXA-48-positive carbapenem-resistant klebsiella pneumoniae isolates in Istanbul, Turkey. Antimicrob Agents Chemother 52: 2950-2954.

11. Thomas CP, Moore LS, Elamin N, Doumith M, Zhang J, et al. (2013) Early (2008-2010) hospital outbreak of Klebsiella pneumoniae producing OXA-48 carbapenemase in the UK. Intern J Antimicrob agents 42: 531-536.

12. Aktas Z, Kayacan CB, Schneider I, Can B, Midilli K, et al. (2008) Carbapenemhydrolyzing oxacillinase, OXA-48, persists in Klebsiella pneumoniae in Istanbul, Turkey. Chemother 54: 101-106.

13. Gulmez D, Woodford N, Palepou MF, Mushtaq S, Metan G, et al. (2008) Carbapenem-resistant Escherichia coli and Klebsiella pneumoniae isolates from Turkey with OXA-48-like carbapenemases and outer membrane protein loss. Intern J Antimicrob agents 31: 523-526.

14. Castanheira M, Mendes RE, Woosley LN, Jones RN (2011) Trends in carbapenemase-producing Escherichia coli and Klebsiella spp. from Europe and the Americas: Report from the SENTRY antimicrobial surveillance programme (2007-09). J Antimicrob Chemother 66: 1409-1411.

15. Nazik H, Ongen B, Mete B, Aydin S, Yemisen M, et al. (2011) Coexistence of blaOXA48 and aac(6')-lb-cr Genes in Klebsiella Pneumoniae Isolates from Istanbul, Turkey. J Intern Med Res 39: 1932-1940.

16. Labarca J, Poirel L, Ozdamar M, Turkoglu S, Nordmann P, et al. (2014) KPCproducing Klebsiella pneumoniae, finally targeting Turkey. New Microbe New Infect 2: 50-51

17. Nordmann P, Dortet L, Poirel L (2012) Carbapenem resistance in Enterobacteriaceae: here is the storm! Trends Mol Med 18: 263-272.

18. Poirel L, Ozdamar M, Ocampo-Sosa AA, Turkoglu S, Ozer UG, et al. (2012) NDM-1-producing Klebsiella pneumoniae now in Turkey. Antimicrob agents Chemother 56: 2784-2785

19. Gonçalves IR, Ferreira ML, Araujo BF, Campos PA, Royer S, et al. (2016) Outbreaks of colistin-resistant and colistin-susceptible KPC-producing Klebsiella pneumoniae in a Brazilian intensive care unit. J Hosp Infect 94: 322-329. 